\title{
L'expression posturo-mimo-gestuelle des secrets dans le roman autobiographique de Philippe Grimbert
}

\author{
Riham EL KHAMISSY \\ Facultade de Filoloxía \\ Université de Ain Chams (Le Caire, Egypte) \\ rihamelkhamissy@yahoo.fr
}

\begin{abstract}
RÉSUMÉ
Que savons-nous de notre passé, de notre histoire personnelle, de nos origines ? Tout ? Rien ? Il a fallu à Philippe Grimbert 191 pages pour raconter comment il a ausculté sa généalogie, sondé les gestes de ses proches, interprété chaque mouvement afin de mettre la main sur des mystères familiaux et de livrer aux lecteurs son roman : Un secret. Auréolé du prix Goncourt des Lycéens en 2004, du Grand Prix littéraire Wizo ${ }^{1}$ en 2005, Un Secret est une autobiographie gonflée de solitude et de silence, où les mystères s'élucident en empruntant des sentiers qui ne sont pas nécessairement ceux de la parole.
\end{abstract}

Mots-clé: Philippe Grimbert, gestes, langage non-verbal

[Recibido, enero 2011; aprobado, junio 2011]

The expression of secrets in Philippe Grimbert's self-biographic novel

\begin{abstract}
What do we know about our past, our personal history, our origins? Everything? Nothing? It took Philippe Grimbert 191 pages to tell how he had tested his genealogy, probed the actions of his family, played every movement to tell his family secrets and give the readers his novel $A$ Secret. Awarded the Prix Goncourt des Lycéens in 2004, and the Wizo in 2005, A Secret is an autobiography filled with solitude and silence, where the mysteries elucidate by taking paths that are not necessarily those of speech.
\end{abstract}

Keywords: Philippe Grimbert, gestures, non-verbal language

\footnotetext{
${ }^{1}$ Le Prix Wizo a été créé en 1978 pour promouvoir les œuvres littéraires françaises d'intérêt juif. Source : http://www.prix-litteraires.net/prix/400,prix-wizo.html,12/12/2010
} 


\section{Introduction}

La plupart des recherches et des travaux sur la gestualité ${ }^{2}$ ne sont que des dictionnaires, des guides ou des index de gestes. Ces ouvrages se limitent à repérer les gestes courants et à les interpréter en fonction des divers systèmes culturels où ils sont en usage. Ces approches sont intéressantes sur le plan de l'ethnographie de la communication, de la psychologie, de la sociologie ou de la sociolinguistique mais amputent au geste des dimensions sémantiques nouvelles qu'il acquiert à chaque occurrence, lesquelles sont en corrélation directe avec son contexte d'émission et de réception.

Dans le présent article, nous ne proposons pas une marche forcée sillonnant le terrain de la posturo-mimo-gestualité $e^{3}$ dans tous les sens, mais une randonnée sémantico-pragmatique débouchant sur une vue panoramique de l'expression de la gestualité chez Philippe Grimbert dans Un Secret. Certes, le texte de Grimbert, comme toute écriture se proposant de rapporter les gestes, « ne peut les représenter autrement que par les mots» (M-D. Popelard et A. Wall 2003 : 93). Notre objectif est de se doter d'une représentation des ressources non-verbales dont puise l'auteur et de les mettre en relation avec les fins qu'elles servent.

\section{Les gestes techniques ou professionnels}

Les gestes techniques appelés également par Louis Porcher gestes "technologiques" désignent " tous les gestes professionnels liés donc à des nécessités techniques, c'est-à-dire dont la forme est définie fonctionnellement. Le geste est alors instrument et apparemment neutre » (1989: 41). Codé et technique, il fonctionne, comme le précise Roger E. Axtell, «par le biais de conventions préalables, du type de celles utilisées par les pompiers, les metteurs en scène ou les marins » $(1993: 10)$. Ces gestes, souvent restreints à des activités bien précises, rappellent des scénarios connus, mobilisant ainsi, au niveau de l'interprétation, nos compétences encyclopédique ${ }^{4}$ et gestuelle ${ }^{5}$. Pour le gesticulateur, il s'agit

\footnotetext{
${ }^{2}$ Nous nous référons en particulier aux travaux suivants : (G.CALBRIS et J. MONTREDON : 1986) ; (D. MORRIS : 1997) ; (S. SANTI, I. GUAÏTELLA, Ch. CAVÉ et G. KONOPCZYNSKI : 2001).

${ }^{3}$ Terme employé pour la première fois en 1984 par Jacques Cosnier et Alain Brossard dans "Communication non-verbale : co-texte ou contexte ?", in La communication non-verbale. Textes de base en psychologie, éd. Delachaux et Niestlé, Neuchâtel, 1984, p.7. Ce terme est repris par Bogdanka Pavelin qui l'utilise comme hyperonyme désignant « l'ensemble des mouvements et des postures corporels » (2002:9). Elle opte pour cette appellation parce qu'elle unit les trois termes utilisés fréquemment lors des descriptions des comportements non-verbaux, à savoir la posture, la mimique et le geste.

${ }^{4}$ La compétence encyclopédique est «l'ensemble virtuellement illimité de connaissance. Ce savoir encyclopédique varie évidemment en fonction de la société où l'on vit et de l'expérience de chacun. (...) dans la compétence encyclopédique, il y a aussi des savoir-faire, l'aptitude d'enchaîner des actions de manière adoptée à une fin. C'est en particulier le cas des scripts (ou scénarios) qui sont des suites stéréotypées d'actions ». (D. MAINGUENEAU $2000: 28$ ).
} 
d'accomplir un devoir, d'exercer une profession. Or, son flux gestuel reste toujours communicant, étant donné qu' « on ne peut pas ne pas communiquer (J. Corraze 1983 :13). Qu'on le veuille ou pas, même "quand nous refusons la communication, nous sommes contraints de signaler cette disposition par une série de signaux : nous ne répondons pas, nous détournons le regard, le visage, etc. » (J. Corraze $1983: 13)$.

Dans Un Secret de Philippe Grimbert, la gestualité professionnelle que le héros narrateur donne à voir est celle de Louise, vieille amie de la famille. Infirmière-masseuse, elle parvient, grâce à ses gestes méticuleux, à briser le silence et à accueillir les confidences les plus intimes: «Depuis des années, elle écoutait mes parents avec la même attention, laissant courir sur eux ces mains énergiques qui les délestaient de leurs soucis: avec leur fatigue, ils abandonnaient chez elle leurs secrets. » (p.35)

À noter que, gestuellement parlant, la fréquence et la répétition facilitent le tissage des liens interpersonnels : "Régulièrement, sur sa table recouverte d'un drap blanc, elle massait mes parents. » «Une fois par semaine, elle m'injectait des vitamines ou m'asseyait face à l'aérosol. » (p.30)

La récurrence de ce contact, avec les mêmes personnages et à des rythmes réguliers, ne laisse pas indifférents les récepteurs du geste, lesquels confèrent à Louise une place importante dans leur vie. Evoquer la gestualité professionnelle nous conduit à l'interrogation suivante : y a-t-il un regard professionnel ? Dans l'usage, on peut parler d'un regard "d'expert", d'un regard "de photographe". Dans les polars, aucun regard n'est plus menaçant ni plus interrogateur que celui du "détective". Dans l'univers de Grimbert, le seul regard professionnel décrit est celui de la tante Esther: " Ma tante, petite femme rousse à la bouche large et aux yeux verts, avait gardé l'habitude de souligner son regard de tragédienne d'un épais trait de crayon. »(p.64) C'est un prétexte pour signaler aux lecteurs, en filigrane, son métier qui ne figure nulle part ailleurs dans le roman.

\section{Les gestes rituels}

Sous cette appellation, nous plaçons les gestes d'obligation qui sont « imposés par la morale sociale, c'est-à-dire par la gestion de la communication stratifiée, définis en termes d'obligations sociales » (L. Porcher 1989: 42). Ils peuvent être religieux ou séculiers, quotidiens ou occasionnels, publics ou privés (comme les fêtes nationales ou familiales). Nous partons du principe selon lequel il n'existe pas de rituel qui ne prenne pas le "corps" comme support direct ou indirect, soit en tant qu'emplacement de signes ou de marques, soit en tant que source de mouvements.

\footnotetext{
${ }^{5}$ La compétence gestuelle correspond à « la capacité de comprendre les gestes d'une société, et, éventuellement de les produire, de savoir ce qu'ils impliquent, de savoir ceux qui sont adéquats dans telle situation et ceux qui ne le sont pas (...)». (G. CALBRIS et L. PORCHER 1989 : 9).
} 
Dans Un Secret, deux événements ont occasionné l'apparition de gestes rituels : le mariage de Maxime et Hannah et la naissance du héros-narrateur.

La description des cérémonies de mariage occupe à elle seule deux chapitres de la quatrième partie du roman. D'abord, il s'agit du passage du mariage civil au mariage religieux : « Après avoir signé le registre de la mairie ils se rendent à la synagogue. » (p.89) Suivent les célébrations et leurs manifestations gestuelles : « On brise des verres, on fait des moulinets avec les serviettes, les plus vigoureux des hommes portent le marié en triomphe sur un siège. » (p.90)

Le fait le plus marquant dans cette scène est l'absence quasi-totale de discours, qu'il soit rapporté directement ou indirectement. Hormis l'allusion à l'expression de félicitations "Mazel Tov ${ }^{6 "}$ (p.90), c'est la description des préparatifs, des invités et des rituels qui préoccupe le narrateur. Ceci explique en grande partie le silence observé quant aux sermons échangés par les mariés, en faveur de la description des gestes rituels devant suivre : " Maxime et Hannah échangent leurs alliances et s'embrassent sous les applaudissements. Ils s'approchent de la table pour apposer leurs signatures sur le registre. » (pp. 93-94)

Rapporter cette série de gestes et de comportements rituels où tout embrasse littéralement le mariage en bonne et due forme est censé présager un bonheur éternel. Or, comme on n'est pas dans le meilleur des mondes, l'image gestuelle trop parfaite du mariage ne suffit pas pour construire des châteaux en Espagne. Le narrateur donne à voir l'idéal pour nous communiquer que nul ne peut prédire l'avenir d'une relation ni assurer sa réussite. Nous ne saurons nous fier aux apparences souvent trompeuses : les gestes ritualisés, même s'ils sont parfaitement effectués, ne sont qu'un passage obligatoire du mariage religieux. C'est d'ailleurs ce que confirme a posteriori l'histoire du roman.

Parmi les conduites religieuses spécifiques liées à des situations et des gestes bien précis, figure le baptême du héros-narrateur :

Mon baptême avait eu lieu si tard que j'en gardais intact le souvenir : le geste de l'officiant, la croix humide imprimée sur mon front, ma sortie de l'église, serré contre le prêtre, sous l'aile brodée de son étoile, un rempart entre la colère du ciel et moi (p.16).

Dans l'exemple susmentionné, la gestualité est sémantiquement riche. Contrairement aux gestes précédemment envisagés dépeints par la syntaxe verbale, les gestes rituels sont rapportés à travers des structures nominales qui ôtent à l'action toute nuance temporelle. Certes, le baptême a eu lieu dans le passé ("Mon baptême avait eu lieu si tard"), mais les détails gestuels de ce rituel possèdent une rémanence immortelle dans la mémoire déclarative ${ }^{7}$ du héros-

\footnotetext{
6 " « mazeltov! » ou « mazaltov ! (parfois en deux mots : « mazel tov ! » ou « mazal tov ! ) est une interjection en hébreu (מזל טוב!) qui est souvent employée au sein de la communauté juive en lieu et place de « félicitations !

7 Mémoire responsable du rappel et de la reconnaissance des mots et des images. ( $C f$. A. LIEURY $1993: 35)$.
} 
narrateur. Si les gestes du prêtre sont pourvus d'un halo de significations religieuses, le baptême catholique du héros acquiert, au-delà des vertus sacrées, un sens additionnel : il devient la stratégie par laquelle les parents tentent de garder secrète une vérité, à savoir leur judéité. Ayant perdu Simon, le demi-frère aîné du narrateur, ce petit persécuté au cours de l'Occupation, les parents, pour sauver leur fils, ont cru devoir cacher sa véritable identité. Un baptême catholique devient donc nécessaire pour travestir la réalité.

En revanche, il existe un autre acte qui a précédé le baptême et auquel le héros-narrateur fait une allusion rapide, à savoir la circoncision : «La marque indélébile imprimée sur mon sexe se réduisait au souvenir d'une intervention chirurgicale nécessaire. Rien de rituel, une simple décision médicale, une parmi d'autres. » (p.16)

Contrairement à toute attente, ce rituel pratiqué par les juifs pour des motifs culturels et religieux se trouve dépouillé de sa fonction traditionnelle voire miné et banalisé. Sur le plan de la gestualité, rien n'est décrit, tout est tu. Comment Grimbert, qui crie haut et fort sa judéité, minimalise un de ses rituels corporels prétendant qu'il s'agit d'une simple décision médicale ? Est-ce un sujet tabou ? Peut-être... Mais à notre sens, deux arguments peuvent justifier l'attitude de Grimbert : d'abord le héros-narrateur n'a découvert sa judéité qu'à l'âge de quinze ans. Si sa famille lui cachait cette identité religieuse, ils ont sûrement inventé un motif médical pour lui expliquer sa circoncision. En outre, cette pratique est exécutée, chez les personnes de confession juive, le huitième jour de la naissance. Il s'ensuit qu'aucun souvenir ne peut en subsister dans la mémoire du héros. Il est donc évident qu'il ne se souvient d'aucun détail de cet épisode de sa vie.

S'il est difficile aux gestes de mentir, il est encore plus difficile de les retenir car « ils traduisent votre assurance et votre désir à communiquer. Chaque geste doit être assumé, il faut aller jusqu'au bout du geste $»^{8}$. À supposer que nous soyons tentée de juger, nous dirons, sans hésitation, que les gestes irrépressibles sont les "gestes expressifs". Dans le roman de Grimbert, ceux-ci sont les gestesvedettes dont la fréquence est la plus élevée et que le narrateur ne laisse jamais passer sous silence : il les détaille, les décrit, et les commente souvent. C'est ce que nous cernerons de près dans les pages qui suivent.

\section{Les gestes expressifs et réactifs}

Tout être humain projette, inconsciemment, ses propres émotions sur son corps :

Les dispositions psychologiques profondes du locuteur, ses intentions latentes sont susceptibles de transparaitre à son insu à travers ses postures, sa mimo-gestualité et concourent ainsi avec les autres informations visuelles

\footnotetext{
8 Les gestes. Généralité, <http://www.communicationoral.com/geste.htm>, date de consultation $(8 / 4 / 2006)$
} 
(morphotype, vêtement, etc.) à induire chez l'allocutaire une impression permanente ou passagère qui influencera le cours de l'interaction » ( J. Cosnier et A. Brossard 1984 : 14-15).

En évoquant la fonction pragmatique des gestes, Scherer distingue deux sous-fonctions, expressive et réactive. Est appelée "fonction expressive" des configurations posturo-mimo-gestuelles «la transmission de l'information concernant des caractéristiques permanentes telles que l'identité sociale (individualité, sexe, âge, appartenance à un groupe social et personnalité) et concernant des états transmitoires mais relativement durables tels que des émotions, des attitudes et des intentions de comportement»(K.R. Scherer 1984: 87). L'autre fonction pragmatique, dite "fonction réactive" correspond à « la manifestation de réactions très brèves à des émissions ou d'autres comportements d'un partenaire» (K.R. Scherer 1984: 87). Nous nous attacherons donc à la connotation des gestes, c'est-à-dire à l'expression des affects selon Jacques Cosnier (1982 : 276), tout en soulignant leur fonctionnement pragmatique.

Étant une composante de la corporéité au même titre que les composantes gestuelles, le regard dans Un secret est significatif : à partir d'un regard, un noyau de sens germe. Bien plus, dans certains cas, le regard devient opérant et performatif. Nous serons, dans ce qui suit, en quête des valeurs sémantiques et pragmatiques dont sont dotés les regards des personnages de Grimbert.

" Le fait de regarder quelqu'un dans - ou entre - les yeux, ou plus généralement au niveau de la moitié supérieure du visage » (M. Cook $1984: 126)$ dit long sur les impressions, l'attitude et les sentiments du sujet regardant. Si l'on examine les regards des personnages du roman, nous constatons, en un clin d'oeil, leur caractère appréciatif. En voici quelques exemples :

Mon univers se restreignit à notre trio. Le dimanche mes parents retrouvaient leurs amis sportifs, pour des tournois de volley ou tennis. Assis dans l'herbe avec mon stylo, je dévorais des yeux ces corps qui bondissaient, luisants de sueurs sous le soleil, j'enrichissais ma collection d'images. ( p.62)

Mais je ressentais la morsure d'une jalousie féroce lorsque Louise évoquait les traits, le corps si bien dessiné du double parfait de Maxime (Simon, le demi-frère du narrateur), couvé par le regard admiratif de son père. (pp.8182)

Dans les cas susmentionnés, le geste oculaire est doté d'une fonction pragmatique. Dans les deux premiers exemples, il opère comme acte de langage ${ }^{9}$, en

\footnotetext{
9 À noter que les termes "langage" et "discours" ne sont pas commutables dans cette recherche. Nous considérons le geste comme acte de langage ou acte de communication mais non pas comme acte de parole, ni acte de discours. Nous prenons les termes "langage" et "communication" pour hyperonymes englobant les signes verbaux, non-verbaux et paraverbaux.
} 
l'occurrence un appréciatif ${ }^{10}$. Ce caractère performatif du regard est souligné par l'épithète "admiratif" et l'expression "dévorer des yeux".

Le fait d'observer fixement une personne, même si c'est par fascination, peut la mettre mal à l'aise, voire la provoquer, notamment lorsque celui qui scrute est quelqu'un que l'on rencontre pour la première fois. Ainsi, déterminé à attirer l'attention de sa belle-sœur, Tania, dont il vient de faire la connaissance, Maxime fixe celle-ci du regard. La jugeant comme la femme la plus belle qu'il n'ait jamais vue, Maxime entend lui rendre hommage par son regard appuyé :

Maxime traverse ses instants (la cérémonie de son mariage) dans un brouillard. Il tourne la tête vers l'assemblée, pour sourire à tous [...]. Tania est assise à côté de son mari, la tête inclinée. Il fixe quelques secondes celle dont la vision a tout balayé. [...]. Alors il ne voit plus que Tania, quitte la cérémonie, oublie sa famille, ses invités. Il fixe la jeune femme jusqu'à ce qu'elle entende enfin son appel muet et relève la tête. Ses boucles noires glissent sur sa robe, s'ouvrent comme un rideau sur ses yeux. Il soutient son regard, une seconde de trop. [...]. Un peu plus tard, pendant que le timbre grave du cantor résonne sous les voûtes de la synagogue, il lève les yeux vers le balcon où se sont installées les femmes, Tania est au premier rang, paupières baissées. La jeune femme a sans doute oublié son premier regard. Mais il la fixe une fois encore. Elle ouvre les yeux, traversée par le même éclair de surprise. (pp.94-95)

Nous constatons que le regard de Maxime ne s'accroche à celui de Tania que deux fois. Toutefois, il acquiert toute sa portée sémantico-pragmatique de plusieurs facteurs réunis : ce regard, dépourvu de paroles accompagnatrices, équivaut à une sollicitation exigeante et revendicative, à "un appel muet" (selon les termes utilisés par le héros-narrateur), lequel demande à être vu et reconnu comme une tentative de séduction. En outre, la hardiesse et l'impertinence de ce geste oculaire appuyé émanant d'un homme qui est sur le point de se marier, en fait un comportement insolent. D'ailleurs, dans Un Secret, ce geste est assimilé à une insulte adressée à Hannah, la mariée : «Il (Maxime) ne veut pas penser à l'injure qu'il fait à Hannah, ainsi qu'à tous ceux qui sont venus les honorer. » (p.94)

Scruter des yeux le corps d'une femme est une attitude libertine. Le regard d'un homme qui donne libre cours à ses instincts et à ses désirs en observant indiscrètement la silhouette d'une femme autre que son épouse, nous le qualifions de pervers, de pernicieux et de coupable. Ceci est observable lors de la description des vacances passées par la famille en Alsace, notamment dans la scène où Tania, nageuse vouée au culte du corps, fait un plongeon acrobatique :

\footnotetext{
10 Les appréciatifs, dans la classification d'Austin, font partie des verdictifs (Austin $1970: 155$ ), alors que, dans celle de Searle, ils figurent parmi les expressifs (1982:32).
} 
Maxime ne peut détacher ses yeux de la ligne de ces épaules, de cette taille, de ces jambes ciselées. Hannah (femme de Maxime) applaudit avant de chercher le regard complice de Maxime. Elle n'y voit que Tania. Elle connaît suffisamment son mari pour y lire un désir fou, une fascination qu'il ne songe même pas à dissimuler. Jamais il ne l'a regardée ainsi. Elle se tourne vers Esther et Georges : tous ces yeux brillant pour Tania de la même ferveur. Elle ne trouve de soutien que dans le regard de Louise, qui a compris et tente de la rassurer d'un sourire." (Un Secret, p.109)

Le regard admiratif de Maxime rejoint celui d'Esther, de Georges et de Hannah, pour ce qui est de l'éblouissement et de la fascination ("tous ces yeux brillant pour Tania de la même ferveur"). Or, il en diffère par son imprudence et par le "désir fou" qui l'anime. C'est d'ailleurs la raison pour laquelle Hannah, l'épouse de Maxime, ressent une jalousie inégalable : «Jamais il ne l'a regardée ainsi » (p.109). À part Louise, personne ne perçoit la souffrance de Hannah. Audelà des mots, un dialogue de regards mutuels noue une complicité entre Hannah et Louise qui compatit et, de son contact oculaire, réconforte Hannah, et d'un sourire la rassure.

Non moins disert que le regard insistant et impudique s'avère le regard fuyant. La déduction la plus courante à propos de l'esquive systématique du contact visuel consiste à dire que «la personne en question est nerveuse et peu sûre d'elle » (M. Cook 1984 :139). Après l'arrestation de Hannah par les Nazis, Tania n'ose plus s'approcher de Maxime. Cette stratégie d'évitement dit beaucoup sur les émotions de Tania. Le glissement oculaire ("Elle (...) baisse les yeux ") cache sa honte d'avoir trahi Hannah. Dans cette même optique (fuir la honte), Tania est soulagée en apprenant la mort de son mari, Robert. Ce décès l'a exemptée d'affronter le regard du mari trompé : «Lorsque Tania apprend la mort de Robert, elle le pleure à peine, il est déjà si loin. Elle pense même qu'elle n'aura pas à affronter son regard. » (p.157)

Outre les regards positifs admiratifs, figurent, dans Un Secret, des regards noirs, hostiles, terrifiants. Nous retiendrons, à titre d'échantillon, celui de l'officier allemand qui a arrêté Hannah et Simon avant qu'ils aient atteint la zone libre :

L'officier ne réagit pas et plonge son regard dans les yeux des deux femmes (Esther et Louise) après avoir contemplé leurs photographies. Il leur rend leurs papiers, contrôle ceux du passeur puis se dirige vers Hannah qui ne s'est pas détournée de la fenêtre. Une fois près d'elle, il tend une main autoritaire et la jeune femme plante ses yeux dans les siens. Louise et Esther retiennent leur souffle, elles la voient fouiller dans son sac, contempler ses papiers, les poser en évidence sur la table avant d'en sortir d'autres qu'elle tend à l'homme, sans lâcher son regard. Décontenancé, l'officier hausse les sourcils. À peine a-t-il jeté un œil sur le document qu'il aboie un ordre [...]. Simon vient de sortir des toilettes et se précipite vers sa mère [...]. L'homme interroge Hannah du regard. Sans hésiter, d'une voix calme elle répond : "c'est mon fils".( pp.135-136) 
La part que prend le regard de l'officier allemand est un indice majeur de sa visée pragmatique. Sans parole concomitante, ce regard est à double fonction : tantôt il menace ("l'officier (...) plonge son regard dans les yeux des deux femmes"), tantôt il interroge ("L'homme interroge Hannah du regard"). Hannah maintient le contact oculaire ("la jeune femme plante ses yeux dans les siens"), voire fixe l'officier en sortant ses papiers d'identité authentiques qui vont la compromettre. En tant que fugitive, son geste laisse inférer son courage : elle n'a peur ni de l'officier ni du destin effroyable qui l'attend. Sur le plan relationnel - notamment celui des relations verticales ${ }^{11}-$, le regard est un taxème ${ }^{12}$ indéniable de dominance. C'est d'ailleurs ce que confirme Guy Barrier se référant à Zimerman : «En ce qui concerne la dominance, Zimerman constate que les personnes pouvant assumer le regard de leur interlocuteur sont jugées plus ascendantes par les observateurs neutres » $(1996$ :73). En outre, l'insistance du regard est révélatrice d'une détermination inaliénable : Hannah a décidé de commettre un acte de suicide en dévoilant aux autorités allemandes sa véritable identité (et donc sa judéité). Remarquons dans cet exemple, la différence entre la manière dont l'officier regarde les personnes et les objets : à Louise et Esther, il réserve un contact oculaire tenace et fixateur, d'où l'emploi réussi du verbe "plonger", et aux objets (photographies et documents), il lance des regards contemplateurs ("après avoir contemplé leurs photographies") ou rapides ("jeter un coup d'œil sur les documents") dans lesquels aucune nuance de violence ni d'agression n'est perçue.

\section{Les gestes extra-communicatifs}

Les émissions non-verbales extra-communicatives sont désignées ainsi, non pas parce qu'elles ne communiquent aucun message mais parce qu'elles «n'ont pas de fonction explicite vis-à-vis des six éléments officiels du système de Shannon Jackobson » (J. Cosnier 1982 : 272). Cosnier ne manque pas d'énumérer quelques traits définitoires des extra-communicatifs en précisant «(a) qu'ils ne surviennent pas au hasard et donc que leur occurrence dépend du processus communicationnel, b) que leur occurrence apporte une information implicite qui peut influencer plus ou moins le processus interactionnel (par exemple quand un des locuteurs se met à bâiller ...), c) qu'enfin, bien qu'en apparence aléatoires et non-significatifs, un certain nombre d'entre eux sont codifiés dans leur usage et dans leur forme » (J. Cosnier $1982: 272$ ).

À ces aspects, Jacques Corraze ajoute trois critères auxquels nous devons la spécificité de ces gestes : 1) Ils ne servent pas la communication mais les besoins intrapsychiques (réduction d'excitation, inhibition d'idée, d'attitude, de

\footnotetext{
11 "La relation verticale correspond à ce qui est connu sous les appellations suivantes :"pouvoir", "rang", "autorité", "dominance ou domination" (vs soumission ou bien encore "système de place". (C. KERBRAT-ORECCHIONI 1992 :72).

${ }^{12}$ Marqueur de relation verticale. (C. KERBRAT-ORECCHIONI 1992 :74).
} 
pulsion); 2) Ils échappent largement au contrôle de la conscience ; 3) Ils sont rares en public où le plus souvent ils sont inhibés ou réduits, fragmentés (1983:178).

Support de signification, le geste centré sur soi est le plus souvent le miroir des émotions et des sensations qui travaillent son producteur : qu'il soit anodin ou nerveux, passager ou répétitif ou un refrain gestuel selon Messenger (2006, 15), qu'il s'agisse d'un geste minime, d'un mouvement léger ou d'une activité motrice largement perçue, le geste auto-centré (ou self-adaptateur) dispose d'une logique tacite consciente ou inconsciente (du côté du gesticulateur) doublée d'une valeur informative irremplaçable. À considérer l'exemple suivant où Tania, la jeune sportive, perfectionne apparemment ses plongeons et procède à une remise à niveau de sa performance: « Elle nage d'une rive à l'autre jusqu'à n'en plus pouvoir, elle parfait ses plongeons depuis la pile du pont effondré. » (p.145)

Tenant compte du contexte où Tania effectue cet exercice sportif violent (la disparition de Hannah et Simon, supposés exécutés par les Allemands), son geste auto-centré acquiert d'autres interprétations potentielles : soit que Tania se sente coupable d'avoir cherché à jouer avec le désir de Maxime en l'absence de sa femme ; alors elle recourt à une auto-punition qu'elle déverse sur son corps. Soit qu'elle aime fortement Maxime, mais sachant qu'il ne pourra pas répondre à sa passion tant qu'il est en deuil, elle épuise son corps pour combattre ses idées impures.

S'avèrent encore plus tangibles les gestes appuyés aux objets (objetsadaptateurs). Guy Barrier avance des exemples d'objets servant de point d'appui comme les bords externes du bureau, accoudoirs de fauteuil, fil de micro, blocnotes (1996 : 68). Exécutés inconsciemment dans la majorité des cas, ces gestes transforment les objets en déversoirs incontournables d'émotions. Leur fonction est avant tout psychologique comme en témoigne l'exemple suivant: «Elle (la tante Esther) fumait cigarette sur cigarette, attendant la clientèle dans leur boutique de nouveautés, près du métro Charonne ». (p.64) Dans l'exemple cité, aux valeurs sémantiques attribuées à l'action de fumer s'ajoute une autre d'ordre pragmatique liée au contexte de réalisation du geste, à savoir l'expression temporelle : le temps qui coule dans l'attente de la clientèle est ample et lent. Cela étant, la réalisation gestuelle souligne à la fois l'intervalle temporel séparant les visites des clients et le caractère anxieux de la tante Esther.

Il convient d'ajouter que l'on peut s'agripper à un objet comme à une bouée de sauvetage ou à une planche de salut. Dans ce cas, l'objet support de nos gestes devient un soutien moral, un rempart qui protège la personne des malheurs qui viennent de fondre sur elle. Raison pour laquelle Hannah, pour éviter de s'affaler, s'accroche au comptoir :

Tania est là-bas, auprès de Maxime. Tout bascule, la vie lui (à Hannah) devient insupportable. Esther et Louise la voient défaillir, ses jambes ne la soutiennent plus, livide elle s'accroche à un comptoir. En lisant la lettre (de Maxime), toutes deux comprennent. (p.130) 
Situons l'exemple dans son contexte : Hannah est bouleversée en apprenant que sa rivale Tania rejoindra Maxime à Saint-Gaultier, le refuge qui abrite la famille juive. Sous l'effet du choc émotif, les jambes de Hannah ne tiennent plus. Sur le point de s'effondrer, elle s'agrippe au comptoir. Ne retrouve-t-on pas ici que ce geste sauve Hannah de l'écroulement ?

A la différence de l'exemple précité, la fonction sécurisante de l'objet pourrait être uniquement d'ordre psychique. Autant dire, l'objet n'a effectivement aucun pouvoir de protection, aucune vertu salvatrice. L'exemple qui saurait le mieux illustrer cette idée est celui du héros-narrateur, serrant contre lui le nounours découvert par sa mère en rangeant la chambre de services : " La peluche râpée, le museau poussiéreux, il était vêtu d'un manteau de tricot. Je m'en étais aussitôt emparé et l'avait serré sur ma poitrine $[\ldots]$ » (p.13)

Ce geste enfantin très répandu et apparemment anodin est typique de la fonction sécurisante réciproque : l'enfant serre son peluche non seulement parce qu'il l'aime, mais aussi parce qu'il imite le geste maternel (la mère embrasse et serre son fils). Ce faisant, il croit procurer à l'objet la tendresse et la protection. Réciproquement, en prenant dans ses bras le nounours, le petit n'est plus seul. Il est réconforté par le fait d'être accompagné, ne fut-ce que par un jouet. La fonction sécurisante de l'objet s'avère donc parfaitement assumée.

La richesse des extra-communicatifs dans le roman de Grimbert devient plus perceptible avec les gestes dirigés vers autrui (les hétéro-adaptateurs). Ceux-ci sont importants à plus d'un titre : ils mettent en présence deux ou plusieurs acteurs. Ils sont donc interactionnels. Qui dit "interaction" dit "relation", qu'elle soit conflictuelle ou coopérative. De surcroît, l'autre est impliqué par mon geste soit parce que nous faisons le geste ensemble ("geste conjoint") (M-D. Popelard et A. Wall $2003: 18$ ), soit parce que mon geste est dirigé vers l'autre ou a une influence sur lui ("geste à effet conjoint") (M-D. Popelard et A. Wall $2003: 18)$.

Le geste maternel est un emblème de la gestualité adaptée à autrui :

Son tempérament (le tempérament de Hannah) ne l'a jamais poussée au combat, aussitôt elle voudrait disparaître, s'effacer pour leur laisser (à Tania et Maxime) la place. La journée s'assombrit, elle (Hannah) va passer le reste de l'après-midi auprès de Simon, l'embrassant, le serrant dans ses bras, plus proche que jamais de son fils. (p.110)

Ne pouvant rivaliser avec Tania la sportive accomplie, Hannah trouve un refuge au contact de son fils. Préférant la passivité à la défense active, elle se laisse vaincre facilement. Elle comble son fils d'amour et de gestes maternels pour compenser son échec à retenir l'attention de son époux. Si le hérosnarrateur nous met au courant des gestes de Hannah envers Simon, il s'interroge sur ceux que sa mère, Tania, vouait à celui-ci : «Avant de devenir ma mère, Tania avait été sa tante. Comment l'appelait-il, quels gestes avait-elle pour lui? » (p.79). En dépit du manque de signes d'affection dans la relation père/fils 
(Maxime/le héros) dans Un Secret, un exemple d'accolade virile mérite d'être traité :

Au moment où je partais me coucher il m'a arrêté, d'une pression légère de sa main sur mon épaule. Je l'ai serré dans mes bras ce que de ma vie je n'avais encore jamais fait (...) Me sentant étrangement fort, je n'ai pas versé une larme, la mort de notre chien avait été l'occasion d'un nouveau retournement : je venais de délivrer mon père de son secret. (p.179)

Ici, le père s'auto-culpabilise pour la mort du chien Echo, comme il l'a déjà fait silencieusement pour le décès de son fils Simon dont l'existence et la disparition constituent le grand secret de la famille. Pour soulager son père, le héros lui raconte qu'il est au courant de sa judéité et de l'exécution de Simon. Il lui dit des propos apaisants pour le libérer du fardeau de remords qu'il portait depuis des années et pour le délivrer du poids du secret qui l'étouffe. Après un laps de temps, le père, reconnaissant, tapote sur l'épaule de son fils qui, ému, l'embrasse. Cette accolade inaugure une nouvelle phase dans la relation père/fils, basée sur la transparence, la confiance, la compréhension et surtout le respect. Le héros ne vivra plus dans l'ombre d'un frère brillant avec qui il est contraint de rivaliser nuit et jour pour l'égaler.

De toutes les réalisations posturo-mimo-gestuelles dirigées vers autrui, celles concernant les relations amoureuses sont les plus fréquentes dans Un Secret. Nous avons déjà vu comment les regards trahissent la passion de Maxime envers Tania. La récurrence de ces regards, leur durée et leur réciprocité en font des signes avant-coureur d'une liaison aventureuse : «Jour après jour les yeux de Maxime se font plus insistants, elle (Tania) leur répond, se laisse envahir par ces vagues de désir. » (p.141)

Dans l'extrait suivant, la déclaration d'amour mutuel se fait uniquement par la voie gestuelle :

Sans échanger un mot, ils (Maxime et Tania) traversent le parc, franchissent le portail qui mène à la berge, étendent leurs serviettes sur l'herbe tiède. Tania se déshabille devant lui, fait glisser sa robe et apparaît en maillot noir qu'elle portait à l'Alsacienne. Elle plonge aussitôt et commence sa traversée, battant l'eau d'un mouvement régulier. Maxime regarde s'éloigner la silhouette de la nageuse qui fend l'eau scintillante. À mi-chemin elle s'arrête pour grimper sur la pile du pont, lui adresse un signe. [...] Face à lui elle reste immobile ruisselante. Elle tend sa main mouillée, il s'en saisit et y enfouit son visage. Elle s'approche de lui, il entoure sa taille de ses bras et appuie sa joue sur l'étoffe du maillot. Il touche enfin le corps de Tania. Après s'être allongé tant de fois en rêve dans sa chaleur c'est la peau glacée de la nageuse qui s'offre à lui. L'eau de la Creuse se mêle à ses larmes. Ils restent ainsi un long moment puis se détachent toujours sans un mot. Tania s'allonge à côté de lui et tous deux fixent le ciel. (pp.147-148) 
Il s'agit là de la déchéance, de la fin d'un combat aussi vieux que le monde entre l'amour et le devoir : Maxime, l'homme en deuil qui succombe à ses désirs, et Tania, la femme mariée qui finit par compatir à la douleur de ce dernier et ne résiste plus à la séduction. Philippe Grimbert donne donc la parole aux gestes qui forment un espace de communication et tiennent lieu de langage intime entre les deux amants.

Analysons le parcours que suit la gestualité dans l'exemple : voir Tania en maillot de bain éveille les aspirations refoulées de Maxime qui fond en larmes, tiraillé entre son deuil et son désir. Pour la première fois, le toucher, longuement réprimé, vient se substituer au dialogue des regards. Tania tend la main à Maxime, comme pour le sauver de ses malheurs et ce dernier y enfonce le visage. Le premier frôlement commande la transformation de leur relation. Une fois le seuil du toucher franchi, les gestes prennent plus d'ampleur : Maxime serre Tania. La distance physique se réduit, entraînant ainsi une grande proximité sur le plan relationnel. Cette intimité ne saurait rester dans le noir. L'amour caché se dévoile progressivement :

Mon père et ma mère, coupables aux yeux de tous, déchirés par leur désir, avaient-ils osé s'aimer au grand jour, se promener main dans la main sur les rives de la Creuse, afficher leur liaison aux yeux de leur famille ? Peu à peu sans doute, avec d'infimes gestes au début, puis s'enhardissant au fil du temps. (p.154)

Les étreintes de Maxime et Tania, considérées successivement comme "insulte" et "crime", soulèvent une question importante, celle du décodage des gestes. Isolée de son contexte de réalisation, l'étreinte dans la culture française n'a généralement rien d'obscène. En revanche, son interprétation diffère lorsqu'elle est replacée en situation. Il s'agit en l'occurrence d'une erreur gestuelle qui équivaut, selon Esther, à l'insulte sur le plan verbal, et au crime sur le plan moral. Cela étant, les interactants co-producteurs de ce geste en sont tenus responsables et doivent en subir les conséquences. Nous pensons avec Louis Porcher que le gesticulateur doit « payer le prix des gestes qu'il fait puisqu'ils sont totalement à lui, et qu'il en est maître » $(1989: 22)$. Il s'ensuit que Maxime et Tania sont contraints de supporter les regards accusateurs, l'indignation et le mépris de leur entourage familial.

Il convient de signaler que l'expression gestuelle est en grande partie déclenchée par notre perception. Le canal visuel collecte des informations renseignant sur le contexte de communication. Tout ce qui entre dans le champ visuel des personnages peut affecter positivement ou négativement leur interaction, voire déclencher une forte émotion qui pourrait s'extérioriser par un flux verbal et/ou posturo-mimo-gestuel. Pour donner corps à ce que nous venons de dire, examinons l'extrait suivant où le héros-narrateur décrit sa réaction gestuelle suite à la projection d'un documentaire sur la Shoah : 
J'ai ri jusqu'à la nausée. Soudain, mon estomac s'est retourné, j'ai cru que j'allais vomir et sans prendre le temps de réfléchir je l'ai frappé (le capitaine de l'équipe de football) violemment au visage. [...] Je l'ai attrapé par les cheveux pour lui cogner la tête contre le sol, j'ai enfoncé mes doigts dans ses yeux, j'ai craché dans sa bouche. (pp.71-72)

Ignorant jusqu'alors sa judéité, le héros raconte comment, à l'âge de quinze ans, il s'est comporté face à une scène de torture filmée, scène qui a fait l'objet de moquerie de son camarade. Nous repérons deux réactions différentes face au même stimulus (l'épisode filmé) : d'une part celle du camarade, réaction verbale enveloppée de moquerie et de médisance ("Ach! Chiens de Juifs" (p.71)), et d'autre part celle du héros, d'abord réaction paraverbale - le rire ("J'ai ri jusqu'à la nausée")-, puis gestuelle, à savoir une rixe.

Notre analyse des extra-communicatifs dans Un Secret nous conduit au constat suivant : les personnages de Grimbert, sous l'effet d'une émotion, manifestent ce qu'ils ressentent soit sur leur corps soit sur les objets qui se trouvent à leur portée, mais rarement sur autrui. L'autre n'est visé par un extracommunicatif que dans une intention relationnelle; dans la majorité des cas, ce geste est consensuel et réciproque.

\section{Conclusion}

Tout au long de cette recherche, notre travail consistait à cibler les signaux d'origine cinétique qui constituent le flux posturo-mimo-gestuel proprement dit dans un Secret. En peignant les gestes, Philippe Grimbert les reconstruit sans maquillage, sans artifice. Grâce à ce roman, l'auteur répond bel et bien à la question suivante : "comment donner forme au non-dit par la description des gestes Tourné vers un passé dont il rapporte les épisodes les plus importants, il évoque la découverte de sa judéité, de son demi-frère, de la Shoah qui a précédé sa naissance, relate l'histoire d'amour qui a réuni ses parents et décrit son baptême catholique et tant d'autres mystères. Bien que les émotions se démasquent habituellement à travers les modes verbaux et paraverbaux, Grimbert a préféré rapporter leurs traces non-verbales, plus justes, plus honnêtes et indétachables de leur contexte d'émission. Nous ne découvrons pas au fil de la lecture un secret mais des secrets. Que les personnages croient les avoir tus sans pour autant se rendre compte que leurs gestes les trahissent.

\section{Bibliographie}

\section{Corpus}

GRIMBERT, Ph. 2004, Un Secret, Paris : Grasset et Fasquelle, Paris, 2004, (191 p.) 


\section{Ouvrages de pragmatique}

\section{a) Ouvrages consacrés à la posturo-mimo-gestualité}

AXTELL, R. E.1993, Le Pouvoir des gestes, Paris : Interéditons.

BARRIER, G.1996, La communication non-verbale. Aspects pragmatiques et gestuels des interactions, Paris : ESF éditeur, coll. Formation permanente en Sciences Humaines.

CABANA, G.2003, Attention! Vos gestes vous trahissent, Québec : Quebecor.

CALBRIS, G. et MONTREDON, J. (éds) 1986, Des gestes et des mots pour le dire, Paris : Clé international.

CORRAZE, J. 1983 (1980), Les communications non-verbales, Paris : Presses Universitaires de France, ${ }^{2 e ̀ m e}$ édition revue et augmentée.

COSNIER, J. (éd.) 1982, Les voies du langage. Communications verbales, gestuelles et animales, Paris : Dunod.

COSNIER, J. et BROSSARD A. (éds) 1984, La communication non-verbale. Textes de base en psychologie, Neuchâtel : Delachaux et Niestlé.

MESSINGER J. 2006, La Grammaire des gestes, Paris : Flammarion.

MORRIS D. 1997 (1994), Le langage des gestes, Paris : Marabout (traduction française par Edith Ochs. Titre original Body Talk, Londres: Jonathan Cape).

PAVELIN, B. 2002, Le geste à la parole, Toulouse : Presses Universitaires du Mirail.

POPELARD, M-D et WALL, A (éds). 2003,, Des faits et des gestes, Bréal : Bréal, coll. Langage et communication.

PORCHER,L.et CALBRIS, G. 1989, Geste et communication, Paris : Hatier/Crédif, coll. LAL.

SANTI S. et al. 2001, Oralité et gestualité. Interactions et comportements multimodaux dans la communication, Paris : L'Harmattan.

\section{b) Articles d'ouvrages collectifs consacrés aux gestes :}

COOK, M. 1984, "Regard et regard réciproque dans les interactions sociales", in La communication non-verbale. Textes de base en psychologie, Neuchâtel : Delachaux et Niestlé, 125-144.

COSNIER, J. 1982, "Communication et langages gestuels", in Les voies du langage. Communications verbales, gestuelles et animales", ouvrage collectif sous la direction de J. Cosnier, éd. Dunod, Paris, 1982, 255-303.

SCHERER K-R.1984, "Les fonctions des signes non-verbaux dans la conversation, in La communication non-verbale. Textes de base en psychologie, Neuchâtel : Delachaux et Niestlé, 71-100. 


\section{c) Ouvrages généraux}

AUSTIN, J- L. 1970 (1962), Quand dire c'est faire, Paris : Seuil (traduction française et introduction de Gilles Lane titre original How to do things with words, Oxford University Press, 1962).

KERBRAT-ORECCHIONI, C. 1992, Les interactions verbales, Tome 1, Paris : Armand Colin.

MAINGUENEAU, D. 2000, Analyser les textes de communication, Paris : Nathan Université, coll. Sup.

SEARLE J-R.1982, Sens et expression, Paris : Minuit.

\section{d) Autres ouvrages}

LIEURY A.1993, La Mémoire, Paris : Flammarion.

LA BRUYÈRE 1968, Les Caractères, préface de Gilbert Sigaux, Lausanne: Rencontre 